\title{
Collaborators for volume 49
}

\section{Axel Ruhe}

Published online: 14 November 2009

(C) Springer Science + Business Media B.V. 2009

We have now completed volume 49 of BIT. It is time to say thank you to all that have helped us making this volume possible.

These are the referees for 2009. Forgive me if I have left out somebody deserving to be on the list. Welcome back to the BIT family next year!

Georgios Akrivis

Andreas Asheim

Domingo Barrera

Zbigniew Bartoszewski

Alfredo Bellen

Peter Benner

Paolo Bientinesi

Sergio Blanes

Carl De Boor

Abderrahman Bouhamidi

John P. Boyd

Claude Brezinski

Luigi Brugnano

Hermann Brunner

Charles Byrne
Franca Calio

Jinhai Chen

Christina C. Christara

Snorre H. Christiansen

Mark W. Coffey

Serge Dubuc

Tommy Elfving

Mark Embree

Terje O. Espelid

Richard S. Falk

Giuseppe Fedele

Bengt Fornberg

Jörg Frauendiener

Stef Graillat

Volker Grimm

A. Ruhe ( $₫)$

Department of Numerical Analysis and Computer Science, NADA, Royal Institute of Technology KTH, 10044 Stockholm, Sweden

e-mail: ruhe@nada.kth.se 
Nicola Guglielmi

Chun-Hua Guo

Ernst Hairer

Marlis Hochbruck

Michiel E. Hochstenbach

Kai Hormann

Weizhang Huang

Daan Huybrechs

Nuutti Hyvonen

Khakim Ikramov

Constantin Ilioi

Arieh Iserles

Artur F. Izmaylov

Tobias Jahnke

Stephen Joe

Klaus Johannsen

Juan Carlos Jorge

Beate Jung

Peter Junghanns

Barbara Kaltenbacher

Misha Kilmer

Peter E. Kloeden

Andrew Knyazev

Lara Yu. Kolotilina

Daniel Kressner

Peter Kunkel

Ming-Jun Lai

Stig Larsson

Lajos Laszlo

Ben Leimkuhler

Ren-Cang Li

Wen Wei Lin

Mingzhu Liu

Anders Logg

Konstantin Makarov

Carla Manni

Francesco Marcellan

Stefano Maset

Francesca Mazzia

Sean McKee
Robert McLachlan

Allen Miller

Giovanni Monegato

Bernard Mourrain

Knut Mørken

S. Nicaise

Erich Nowak

Mario Ohlberger

Brynjulf Owren

César Palencia de Lara

Ola Petri

Rosemary Renaut

Marie Rognes

Christian Rohde

Einar Rønquist

Keijo Ruotsalainen

Paul Sablonnière

Driss Sbibih

Hans Joachim Schroll

Jie Shen

Vasile Sima

Theodore Simos

William Skamarock

Ian Sloan

Tor Sørevik

Daniel B. Szyld

Xue-Cheng Tai

Mechthild Thalhammer

Philippe Toint

Catalin Turc

Guido Vanden Berghe

Christof Voemel

Bruno Welfert

Shufang $\mathrm{Xu}$

Ningning Yan

Qiang Ye 


\section{Introduction to the contents of issue 49:4}

We start this issue with a contribution of Mathematical Physics interest. Snorre Christiansen and Tore Halvorsen study convergence of discretizations to the Maxwell equations of electromagnetism. A lattice gauge theory is shown to preserve important symmetries and its convergence in energy norm is proved.

We have one work in linear algebra. Iveta Hnětynková, Martin Plešinger and Zdeněk Strakoš solve a matrix regularization problem with the quite common technique of first applying the Golub Kahan bidiagonalization algorithm to find a core problem, and then regularizing this low dimensional bidiagonal core problem. A stopping criterium is developed that can be used also when the noise level in the right hand side observation vector is unknown.

Two contributions deal with convergence of finite element approximations. Mohammad Izadi gives estimates for finite element approximations to one-dimensional time dependent coupling equations of two scalar conservation laws. A variant of a streamline diffusion discretization is used.

Ljiljana Teofanov and Helena Zarin study a finite element solution of singularly perturbed elliptic problems with two small perturbation parameters. The solution then has rapid changes in boundary layer parts of the domain. The interest is in superconvergence and postprocessing on a layer adapted mesh.

Finally we have a new contribution to the chebfun system, where functions are represented by interpolation polynomials to machine accuracy. Richardo Pachón and Lloyd Trefethen give an implementation of the Remez exchange algorithm to find a minimax polynomial approximation to a function over an interval. The use of barycentric coordinates and a global rootfinder makes the algorithm usable also for polynomials of very high degrees.

Welcome to another issue of BIT!

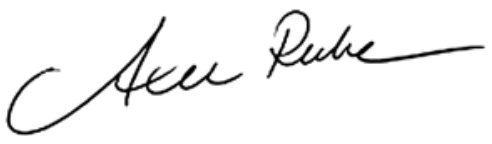

Axel Ruhe 